\title{
DARIO GRAFFI
}

Il 28 Dicembre 1990 si è spento a Bologna Dario Graffi, per lunghi anni membro del Comitato di Redazione della nostra rivista.

Nato a Rovigo il $1^{\circ}$ gennaio 1905, Dario Graffi si laureò presso l'Università di Bologna in Fisica, nel 1925, e in Matematica, nel 1927, riportando in ambedue le lauree il massimo dei voti e la lode. Assistente alla Cattedra di Fisica Tecnica nella Scuola (poi Facoltà) d'Ingegneria di Bologna dal 1925 al 1935, Professore di Matematica e Fisica nel Liceo Classico «Dettori» di Cagliari dal 1935 al 1936, divenne Professore Straordinario di Meccanica Razionale nell'Università di Torino dal 1936 al 1938 e di Bologna nell'anno accademico 1938-39, e fu successivamente Professore Ordinario di Meccanica Razionale presso l'ateneo felsineo il $1^{\circ}$ Novembre 1939. Dal 1980 era Professore Emerito.

Era Socio Nazionale dell'Accademia dei Lincei dal 1966 (dopo essere stato SocioCorrispondente dal 1953), Accademico effettivo (Benedettino) dell'Accademia delle Scienze di Bologna (di cui fu anche Presidente), Socio nazionale dell'Accademia delle Scienze di Torino, Socio onorario dell'Accademia di Scienze, Lettere ed Arti di Modena, Socio Corrispondente dell'Istituto Lombardo di Scienze e Lettere, dell'Accademia Ligure di Scienze e Lettere e dell'Accademia dei Concordi di Rovigo, Socio Ordinario dell'Accademia delle Scienze di Ferrara.

Alla scienza dette cospicui contributi originali in vasti settori di ricerca che riguardano la Meccanica, la Termodinamica e l'Elettromagnetismo. Coi lavori sui fenomeni ereditarî, pubblicati tra il 1928 e il 1935, si colloca tra i precursori di questo campo d'indagini, oggi divenuto intenso oggetto di ricerche. Ma i suoi interessi amplissimi spaziarono dai teoremi di reciprocità nell'elasticità e nell'elettromagnetismo alla meccanica delle masse variabili, dalla termomeccanica dei continui alla propagazione elettromagnetica libera e guidata entro mezzi eterogenei, dalla meccanica delle oscillazioni non lineari alle proprietà degli invarianti adiabatici. Notevole è poi il gruppo dei suoi lavori dedicati ai teoremi d'unicità nella meccanica dei continui e nell'elettromagnetismo.

Lascia un vivo compianto in quanti conobbero e seppero apprezzare le Sue doti d'uomo e di studioso.

Carlo Cercignani 